\title{
Echocardiographic findings associated with mortality or transplant in patients with pulmonary arterial hypertension: A systematic review and meta-analysis
}

\author{
V.J.M. Baggen ${ }^{1,2}$ • M.M.P. Driessen ${ }^{1}$ M.C. Post ${ }^{3}$ A.P. van Dijk ${ }^{4}$ J.W. Roos-Hesselink ${ }^{2}$ A.E. van den Bosch ${ }^{2}$ \\ J.J.M. Takkenberg ${ }^{5}$ G.T Sieswerda ${ }^{1}$
}

Published online: 17 May 2016

(c) The Author(s) 2016. This article is available at SpringerLink with Open Access

\begin{abstract}
Background Identification of patients at risk of deterioration is essential to guide clinical management in pulmonary arterial hypertension (PAH). This study aims to provide a comprehensive overview of well-investigated echocardiographic findings that are associated with clinical deterioration in PAH.

Methods MEDLINE and EMBASE databases were systematically searched for longitudinal studies published by April 2015 that reported associations between echocardiographic findings and mortality, transplant or clinical worsening. Meta-analysis using random effect models was performed for echocardiographic findings investigated by four or more studies. In case of statistical heterogeneity a sensitivity analysis was conducted.

Results Thirty-seven papers investigating 51 echocardiographic findings were included. Meta-analysis of univariable hazard ratios (HRs) and sensitivity analysis showed
\end{abstract}

Electronic supplementary material The online version of this article (doi: 10.1007/s12471-016-0845-3) contains supplementary material, which is available to authorized users.

G.T Sieswerda

G.T.Sieswerda@umcutrecht.nl

1 Department of Cardiology, University Medical Centre Utrecht, 3508 GA Utrecht, The Netherlands

2 Department of Cardiology, Erasmus Medical Centre, Rotterdam, The Netherlands

3 Department of Cardiology, St. Antonius Hospital, Nieuwegein, The Netherlands

4 Department of Cardiology, Radboud University Medical Centre, Nijmegen, The Netherlands

5 Department of Cardio-Thoracic Surgery, Erasmus Medical Centre, Rotterdam, The Netherlands that presence of pericardial effusion (pooled HR 1.70; $95 \%$ CI 1.44-1.99), right atrial area (pooled HR 1.71; $95 \%$ CI 1.38-2.13) and tricuspid annular plane systolic excursion (TAPSE; pooled HR 1.72; $95 \%$ CI 1.34-2.20) were the most well-investigated and robust predictors of mortality or transplant.

Conclusions This meta-analysis substantiates the clinical yield of specific echocardiographic findings in the prognostication of PAH patients in day-to-day practice. In particular, pericardial effusion, right atrial area and TAPSE are of prognostic value.

Keywords Pulmonary arterial hypertension - Prognosis . Echocardiography $\cdot$ Mortality $\cdot$ Non-invasive imaging

\section{Introduction}

The ongoing research on pulmonary arterial hypertension (PAH) has led to increased awareness of the pathophysiological, haemodynamic and clinical consequences of this devastating disease [1]. Without intervention, progressive remodelling of the distal pulmonary arteries leads to elevated pulmonary vascular resistance, eventually resulting in right heart failure and death $[1,2]$. Fortunately, advances in therapeutic modalities have greatly improved the survival and quality of life in patients with PAH [3]. However, the natural course of the disease varies widely between individuals, as some patients live for decades while others die within months of diagnosis [4]. In order to guide optimal clinical management, it is therefore essential to accurately monitor disease progression and estimate prognosis in PAH.

Previously reported predictors of mortality include aetiology of PAH, gender and several functional, haemodynamic and biochemical variables [5-8]. Echocardiogra- 


\section{Advertisement placed here.}

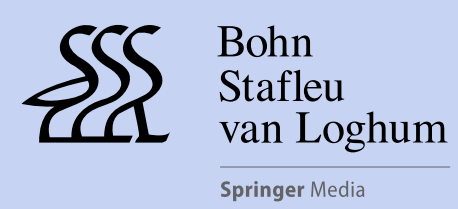

Houten 2016 


\section{Advertisement placed here.}

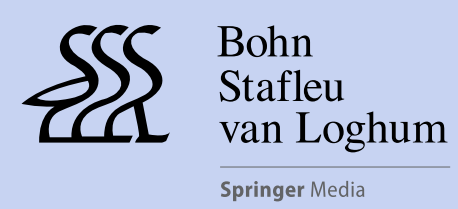

Houten 2016 


\section{Advertisement placed here.}

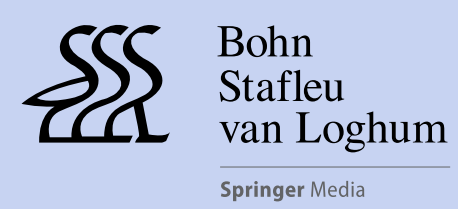

Houten 2016 


\section{Advertisement placed here.}

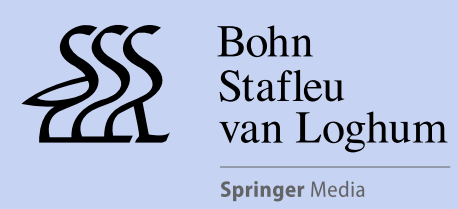

Houten 2016 
Fig. 1 PRISMA 2009 flow diagram

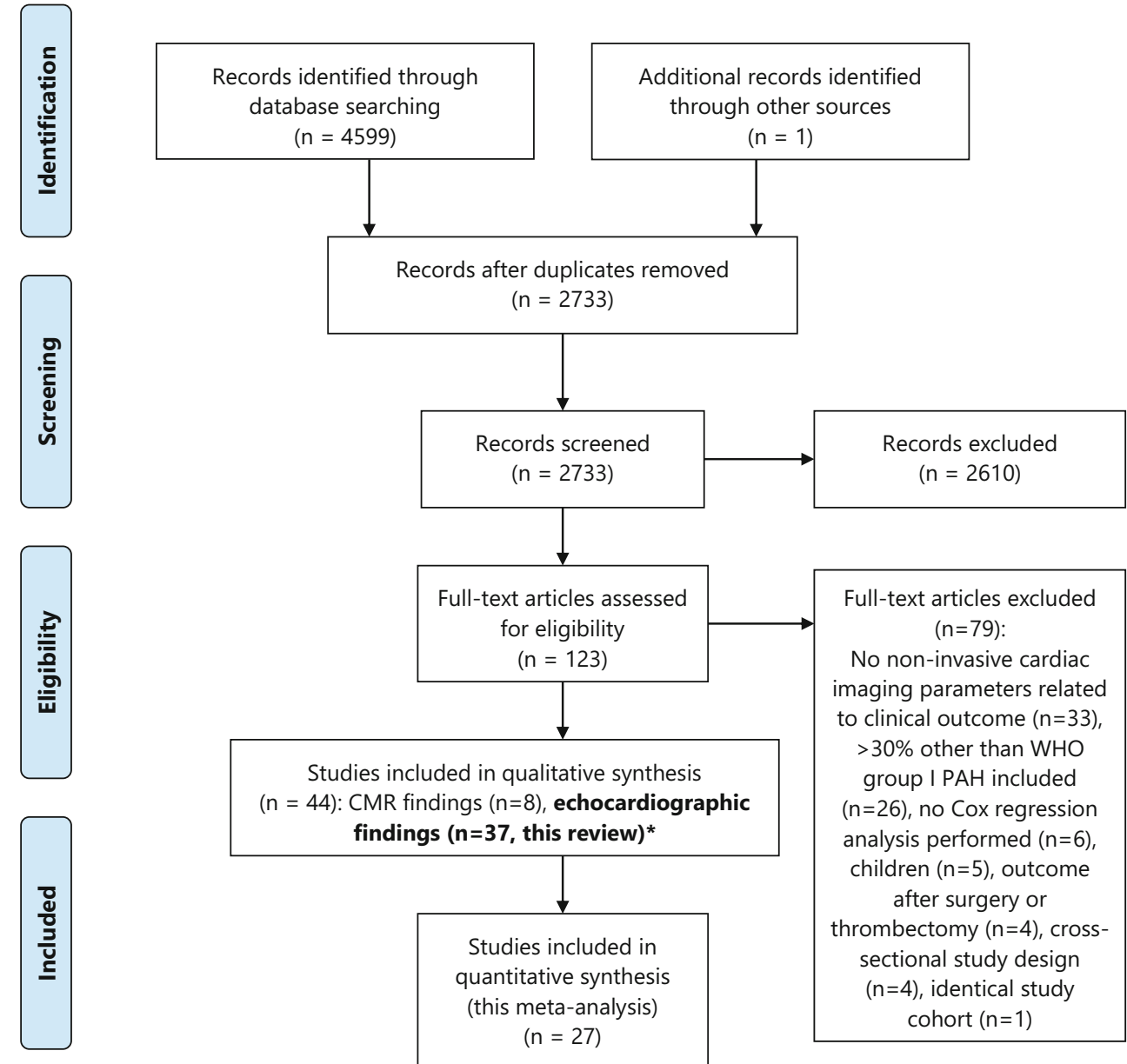

*one study investigated both echocardiographic and CMR findings.

From: Moher D, Liberati A, Tetzlaff J, Altman DG. The PRISMA Group (2009). Preferred Reporting /tems for Systematic Reviews and MetaAnalyses: The PRISMA Statement. PLoS Med 6(6): e1000097. doi:10.1371/journal.pmed1000097

For more information, visit www.prisma-statement.org phy is the most readily available cardiac imaging modality and is universally used in the follow-up of patients with PAH. Current literature reports several echocardiographic findings that may provide important prognostic information. The goal of this study is to provide a comprehensive overview of the most thoroughly investigated baseline echocardiographic findings that are associated with adverse clinical outcome in PAH. Separately, this study evaluates the prognostic value of a change in echocardiographic findings during a follow-up period.

\section{Methods}

This systematic review was conducted in accordance with the PRISMA statement [9]. A pre-defined review protocol, as adopted by this study, can be accessed through PROSPERO (registration number: CRD42014009231).

\section{Literature search strategy}

A comprehensive systematic search was performed in MEDLINE (via PubMed interface) and EMBASE electronic databases on 29 April 2015 using combinations of all synonyms for: $\mathrm{PAH}$, echocardiography and relevant clinical outcomes (components of the Dana Point Time To Clinical Worsening composite endpoint) [1]. A validated prognostic search filter with the highest sensitivity $(98 \%)$ was added to the search syntax [10]. No language or publication period restrictions were applied. The full original search syntax is provided in Supplementary File 1. 


\section{Selection of papers}

A flow diagram of the selection process is shown in Fig. 1 [9]. After deduplication, one author performed screening and selection of articles based on title and abstract, using the following exclusion criteria: inappropriate study type (cross-sectional or trial design, reviews, case reports with $<10$ patients, editorials or congress abstracts), nonclinical data (technical, animal and in-vitro studies), study population without PAH (e. g. acute pulmonary embolism, exercise-induced pulmonary hypertension), studies that included children $<12$ years, and studies that did not relate echocardiographic findings to clinical outcome. Full-text screening was performed by two authors; reasons for exclusion are described in Fig. 1. All references of the excluded reviews and included articles were cross-checked to identify possible relevant articles missed in the original search syntax.

\section{Assessment of methodological quality}

Study quality was critically appraised using previously developed criteria for prognostic studies [11]. We assessed study design, missing data and loss to follow-up (selection bias), adequate description and measurement of imaging features and outcome (information bias), reported effect size, treatment of continuous risk predictors and multivariable adjustment for possible confounders.

\section{Data extraction and analysis}

Study characteristics and hazard ratios (HRs) for all investigated echocardiographic findings with accompanying $95 \%$ confidence intervals were extracted using a standardised form. Meta-analysis was performed for all echocardiographic findings that were investigated as continuous parameters by four or more studies, using random effect models. In order to unify the extracted data to allow more studies to be pooled, HRs were recalculated to one uniform clinically applicable number of units change. Heterogeneity was assessed using Cochran's Q test and the $\mathrm{I}^{2}$ statistic. Imaging findings investigated as dichotomous variables were additionally presented at the bottom of the corresponding forest plots. For all echocardiographic measurements with significant heterogeneity $\left(\mathrm{I}^{2}>50 \%\right.$ or Cochran's Q $p$-value $<0.10)$ a sensitivity analysis was performed by excluding specific patient subgroups.

If study data were used in multiple papers and the same echocardiographic findings were evaluated, only the study with the largest sample size was used to exclude the risk of using duplicate data in our meta-analysis. The risk of publication bias was assessed using visual inspection of funnel plots and Egger's test.

\section{Results}

\section{Search results}

The systematic literature search in MEDLINE and EMBASE and extensive reference cross-checking retrieved 2,733 potentially relevant records, of which 2,610 were excluded based on title and abstract (Fig. 1). After full-text review of the remaining 123 articles, 37 papers were finally selected [6, 12-47]. Study and patient characteristics of the included studies are shown in Tab. 1.

The total number of patients per study ranged from 25 to 2,716, with a mean age ranging from 31-61 years (60-98\% female) and 35-100\% of patients in New York Heart Association (NYHA) class III-IV. Twelve studies included patients with congenital heart disease (CHD) (6-100\% of patients) $[6,22,25,30,31,33,36,41-43,46,47]$ and seven studies included a subset of patients with pulmonary hypertension group III or IV ( $<30 \%$ of total study population) $[18,20,29,33,35-37]$. The majority of studies used death or transplant as primary outcome; only five studies (14\%) used a composite outcome, additionally including hospitalisation for heart failure [20, 35, 41, 47], need for a second vasodilator drug or worsening of functional class [36]. Mean follow-up duration varied between 11 and 53 months, with the primary outcome event occurring in $6-340$ patients (10-62\% of study population).

\section{Methodological aspects}

In Fig. 2, an overview of the methodological quality of all included studies is presented. Individual bias assessment per study is provided in Supplementary File 2. Studies prospectively included consecutive patients diagnosed with the disease ( $43 \%$ of studies) or retrospectively reviewed echocardiographic images. Information on missing values and loss to follow-up was not reported in $16(43 \%)$ and $21(57 \%)$ of the 37 studies, respectively. It is therefore important to recognise the possible impact of selection bias on individual study outcomes.

All studies used right heart catheterisation for the diagnosis of PAH in $100 \%$ of the included patients, except for one study that used right heart catheterisation in $87 \%$ and echocardiography in $13 \%$ of patients [35]. Definition and measurement of echocardiographic findings and study outcome was appropriate and consistent in the majority of studies, therefore the impact of information bias is assumed to be small.

Cox regression analysis was performed in all studies; however large differences for predictors included in the multivariable analysis were found. Twenty-nine studies performed some form of multivariable adjustment, of which only 15 adjusted for age and gender. Only nine studies 
Tab. 1 Study characteristics

\begin{tabular}{|c|c|c|c|c|c|c|c|c|c|c|c|c|c|c|}
\hline $\begin{array}{l}\text { Study, } \\
\text { ref } \\
\text { no }\end{array}$ & $\begin{array}{l}\text { Size, } \\
n\end{array}$ & $\begin{array}{l}\text { Age, } \\
\text { years }\end{array}$ & $\begin{array}{l}\text { Gender, } \\
\% \\
\text { fe- } \\
\text { male }\end{array}$ & $\begin{array}{l}\text { NYHA } \\
\text { class } \\
\text { III-IV, } \\
\%\end{array}$ & $\begin{array}{l}\text { IPAH/ } \\
\text { heredi- } \\
\text { tary }\end{array}$ & $\begin{array}{l}\text { Drug/ } \\
\text { toxin }\end{array}$ & $\begin{array}{l}\text { PAH- } \\
\text { CTD }\end{array}$ & Po-PAH & $\begin{array}{l}\text { PAH- } \\
\text { CHD }\end{array}$ & $\begin{array}{l}\text { WHO } \\
\text { I PAH } \\
\text { (other/not } \\
\text { speci- } \\
\text { fied) }\end{array}$ & $\begin{array}{l}\text { WHO } \\
\text { III } \\
\text { (lung } \\
\text { dis- } \\
\text { ease) }\end{array}$ & $\begin{array}{l}\text { WHO } \\
\text { IV } \\
\text { (CTEPH) }\end{array}$ & $\begin{array}{l}\text { Follow-up } \\
\text { duration, } \\
\text { months }\end{array}$ & $\begin{array}{l}\text { Events, } \\
n(\%)\end{array}$ \\
\hline [11] & 26 & $\begin{array}{l}41 \\
{[16-70]}\end{array}$ & 69 & NR & 100 & - & - & - & - & - & - & - & $24 \pm 14$ & $16(62)$ \\
\hline [12] & 26 & $43 \pm 17$ & 73 & 58 & 100 & - & - & - & - & - & - & - & NR & $6(23)$ \\
\hline [13] & 53 & $45 \pm 14$ & 72 & 70 & 100 & - & - & - & - & - & - & - & $35[\mathrm{NR}]$ & $32(60)$ \\
\hline [14] & 43 & $\begin{array}{l}37 \\
{[14-67]}\end{array}$ & 70 & 86 & 100 & - & - & - & - & - & - & - & $21 \pm 16$ & $12(28)$ \\
\hline [15] & 25 & $38 \pm 13$ & 76 & 100 & 100 & - & - & - & - & - & - & - & $12[0-84]$ & $13(52)$ \\
\hline [16] & 81 & $40 \pm 15$ & 73 & 100 & 100 & - & - & - & - & - & - & - & $36 \pm 15$ & $41(51)$ \\
\hline [17] & 63 & $55 \pm 15$ & 83 & 70 & 37 & - & 38 & - & - & - & 21 & 5 & $19[10-22]^{\mathrm{a}}$ & $23(37)$ \\
\hline [18] & 54 & $52 \pm 11$ & 76 & 76 & 100 & - & - & - & - & - & - & - & $50[\mathrm{NR}]$ & $12(22)$ \\
\hline [19] & 50 & $46 \pm 13$ & 78 & 42 & 46 & - & 22 & - & - & 4 & - & 28 & 14 [12-18] & $19(38)$ \\
\hline [6] & 2716 & $50 \pm 17$ & 79 & 54 & 49 & 5 & 24 & 5 & 12 & 5 & - & - & 17 [0-24] & 340 (13) \\
\hline [20] & 76 & $61 \pm 11$ & 84 & 53 & - & - & 100 & - & - & - & - & - & $\begin{array}{l}36 \\
{[\mathrm{NR}-113]}\end{array}$ & $42(55)$ \\
\hline [21] & 32 & $53 \pm 16$ & 66 & 91 & 69 & 16 & 6 & - & 9 & - & - & - & $21[\mathrm{NR}]$ & $17(53)$ \\
\hline [22] & 59 & $46 \pm 16$ & 63 & 66 & 100 & - & - & - & - & - & - & - & $52[28-79]^{\mathrm{a}}$ & $23(39)$ \\
\hline [23] & 72 & $52 \pm 16$ & 72 & 76 & 100 & - & - & - & - & - & - & - & $38[14-71]^{\mathrm{a}}$ & $22(31)$ \\
\hline [24] & 484 & $52 \pm 15$ & 75 & 71 & 56 & - & 24 & 11 & 9 & - & - & - & 38 [16-60] & $264(55)$ \\
\hline [25] & 50 & $61 \pm 11$ & 98 & 70 & - & - & 100 & - & - & - & - & - & 16 [9-39] & $25(50)$ \\
\hline [26] & 80 & $56 \pm 14$ & 76 & 72 & 43 & - & 41 & 10 & - & 6 & - & - & $24[\mathrm{NR}]$ & $33(41)$ \\
\hline [27] & 95 & $31 \pm 10$ & 64 & 56 & 100 & - & - & - & - & - & - & - & $21 \pm 15$ & $27(28)$ \\
\hline [28] & 57 & $52 \pm 14$ & 28 & 100 & 63 & - & 18 & 11 & - & 5 & - & 3 & $25 \pm 29$ & $29(51)$ \\
\hline [29] & 181 & $39 \pm 13$ & 67 & 67 & - & - & - & - & 100 & - & - & - & 16 [7-46] & $19(10)$ \\
\hline [30] & 154 & $54 \pm 9$ & 84 & NR & 46 & 1 & 40 & 5 & 6 & 3 & - & - & $36[17-71]^{\mathrm{a}}$ & $71(46)$ \\
\hline [31] & 61 & $48 \pm 18$ & 84 & 69 & 100 & - & - & - & - & - & - & - & NR & NR \\
\hline [32] & 142 & $59 \pm 15$ & 65 & 44 & 31 & 9 & 19 & 4 & 9 & 1 & - & 27 & 11 [6-39] & $28(20)$ \\
\hline [33] & 577 & $53 \pm 15$ & 75 & 70 & - & - & - & - & - & 100 & - & - & $57 \pm 50$ & NR \\
\hline [34] & 406 & $59 \pm 16$ & 65 & 46 & - & - & - & - & - & 74 & 14 & 12 & $16[8-20]^{\mathrm{a}}$ & $73(18)$ \\
\hline [35] & 32 & $39 \pm 15$ & 69 & 59 & 22 & - & 16 & - & 53 & - & - & 9 & 14 [8-21] & $15(47)$ \\
\hline [36] & 124 & $54 \pm 16$ & 70 & 92 & - & - & - & - & - & 84 & - & 16 & $36 \pm 22$ & $31(25)$ \\
\hline [37] & 71 & $57 \pm 14$ & 76 & 75 & 46 & - & 41 & 6 & - & 7 & - & - & $24[\mathrm{NR}]$ & $20(28)$ \\
\hline [38] & 50 & $56 \pm 12$ & 84 & 72 & 42 & - & 38 & 14 & - & 6 & - & - & $48[\mathrm{NR}]$ & NR \\
\hline [39] & 102 & $54 \pm 16$ & 84 & NR & 47 & - & 24 & - & - & 29 & - & - & $44[22-79]^{\mathrm{a}}$ & $43(42)$ \\
\hline [40] & 37 & $46 \pm 14$ & 76 & 35 & 65 & - & 5 & - & 24 & 5 & - & - & $16[13-18]^{\mathrm{a}}$ & 7 (19) \\
\hline [41] & 48 & $44 \pm 14$ & 83 & 100 & 67 & - & 21 & 6 & 6 & - & - & - & $53[21-80]^{\mathrm{a}}$ & $18(38)$ \\
\hline [42] & 91 & $42 \pm 14$ & 60 & 73 & - & - & - & - & 100 & - & - & - & $46[4-64]$ & $24(26)$ \\
\hline [43] & 79 & $\begin{array}{l}48 \\
{[24-65]}\end{array}$ & 66 & 92 & 92 & - & 8 & - & - & - & - & - & NR [12-92] & $27(34)$ \\
\hline [44] & 121 & $60 \pm 14$ & 66 & 63 & 39 & - & 36 & 18 & - & 6 & - & - & $37 \pm 36$ & $49(40)$ \\
\hline [45] & 200 & $54 \pm 15$ & 71 & 50 & 47 & 1 & 33 & 7 & 12 & - & - & - & $43 \pm 31$ & $106(53)$ \\
\hline [46] & 51 & $60 \pm 15$ & 73 & 71 & 33 & - & 55 & 6 & 6 & - & - & - & $36 \pm 24$ & $8(16)$ \\
\hline
\end{tabular}

$C H D$ congenital heart disease, $C T D$ connective tissue disease, $C T E P H$ chronic thromboembolic pulmonary hypertension, $H F$ heart failure, $I P A H$ idiopathic pulmonary arterial hypertension, $N Y H A$ New York Heart Association, $P A H$ pulmonary arterial hypertension, $P H$ pulmonary hypertension, $S D$ standard deviation, $N R$ not reported, $W H O$ World Health Organization. ainterquartile range, otherwise reported as median [range] or mean $\pm \mathrm{SD}$ 
Fig. 2 Methodological quality of the included studies. Methodological quality of the included studies was assessed on the following domains of potential bias: completeness of data (selection bias), standardisation of prognostic factors and study outcome (information bias) and statistical calculation of effect size (study outcome)

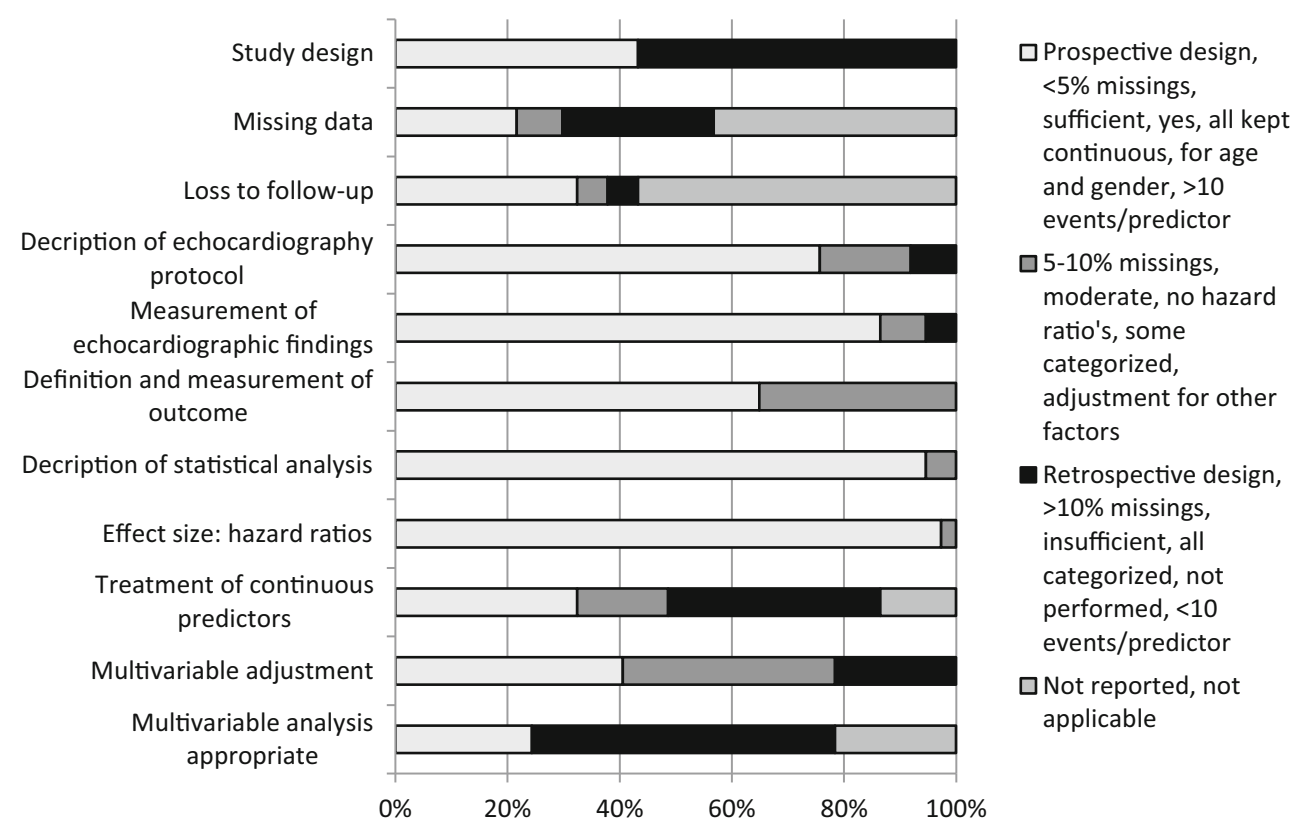

Serial echocardiographic evaluation

(24\%) used more than ten events per predictor. Because of this large variety between studies and overall poor methodological quality of multivariable adjustment, it was chosen to present only the univariable HRs in forest plots.

\section{Prognostic value of baseline echocardiographic findings}

In 37 studies, in total 51 echocardiographic findings were evaluated (Supplementary File 3). Meta-analysis was performed for ten echocardiographic findings that were suitable for pooling of results among four or more studies: presence of pericardial effusion, right atrial area, right ventricular (RV) pressure estimates, severity of tricuspid regurgitation, estimated right atrial pressure, left ventricular (LV) eccentricity index (Fig. 3a), tricuspid annular plane systolic excursion (TAPSE), RV fractional area change, Tei index (an index of RV myocardial performance) and RV free wall longitudinal peak systolic strain (LPSS) (Fig. 3b).

Although not included in the meta-analysis, RV end-diastolic basal dimension $[24,30,33,36]$ or area $[17,25$, $36,37]$ and tissue Doppler velocity (S') of the tricuspid valve annulus [30, 33, 35, 36, 41] were investigated by several studies and could be of prognostic importance. Less investigated echocardiographic measurements such as pulmonary artery capacitance [19, 35], several strain values [27, 38, 44], RV diastolic dysfunction [12, 20, 30], LV end-diastolic volume [23], systolic pulmonary artery pressure increase during exercise [37] and RV load adaptation index [44] seem promising but require further evaluation.
Five studies included in this review investigated the prognostic value of a change in echocardiographic findings during a follow-up period, rather than their absolute baseline values, as indicated in Supplementary File 3 [39, 42, 44, 46, 47]. Patients with $\geq 5 \%$ improvement in RV free wall LPSS on PAH treatment at $6 \pm 2$ months follow-up had a significantly reduced mortality risk at four years (HR 0.13 ; $95 \%$ CI 0.03-0.50) [39]. Tonelli et al. showed that overall mortality was associated with a $10 \%$ increase in RV end-diastolic area (HR 1.37; $95 \%$ CI 1.08-1.75), tricuspid regurgitation velocity (HR 1.72; $95 \%$ CI 1.12-2.70) and difference in qualitative RV function (HR per unit of improvement 0.55 ; $95 \%$ CI $0.31-0.96$ ) at one-year follow-up [42]. Sano et al. recently reported that a mid-term change in RV end-systolic area (HR 0.92; $95 \%$ CI 0.86-0.98) and in right atrial area (HR 0.95; $95 \%$ CI 0.92-0.99) were significantly related to long-term outcome [47]. In addition, changes in RV load-adaptation index and pericardial effusion have been associated with clinical outcomes in PAH $[44,46]$.

\section{Risk of bias assessment}

Heterogeneity statistics (Cochran's Q and $\mathrm{I}^{2}$ ) are presented in the corresponding forest plots in Fig. $3 a$ and b. For all echocardiographic measurements with significant statistical heterogeneity, we performed a sensitivity analysis to evaluate possible sources for heterogeneity (Tab. 2). We excluded specific studies $(<70 \%$ in NYHA class III-IV, $<100 \%$ PAH, inclusion of CHD, $<50 \%$ on PAH medication at baseline, other endpoints than mortality or trans- 
Presence of pericardial effusion

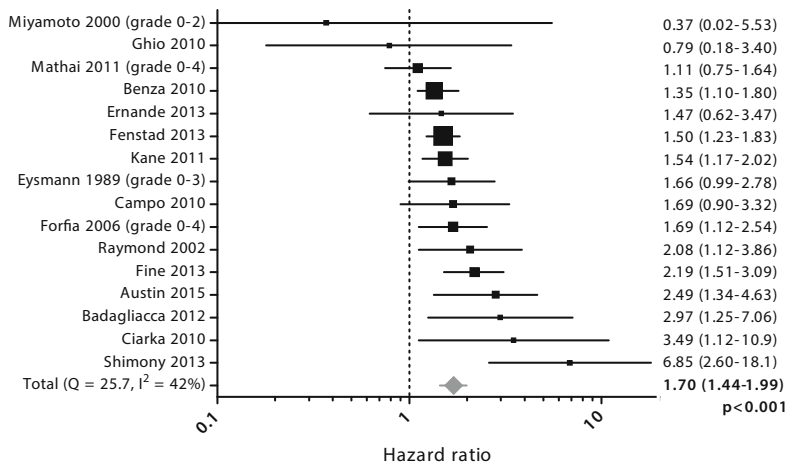

$\mathrm{RV}$ pressure estimates (per $10 \mathrm{mmHg}$ increase)

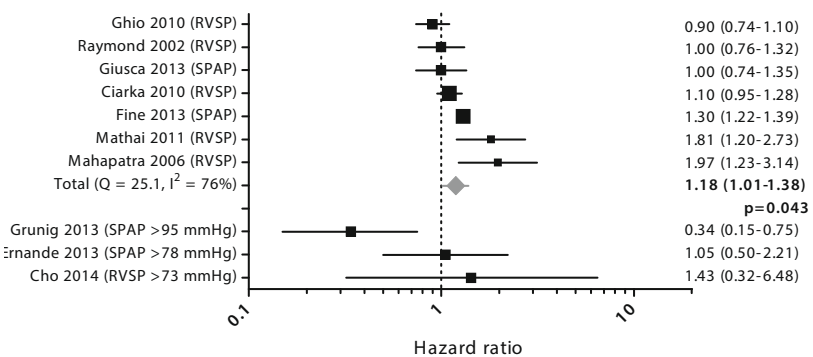

Estimated RA pressure

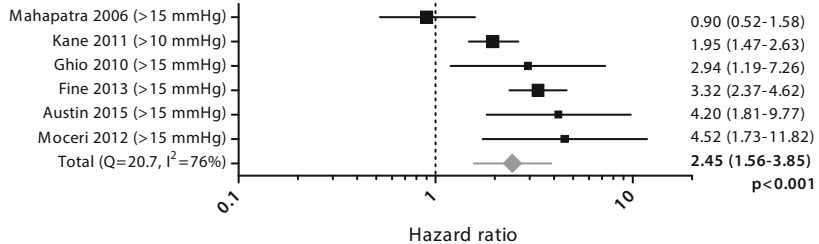

a

TAPSE (per $5 \mathrm{~mm}$ decrease)

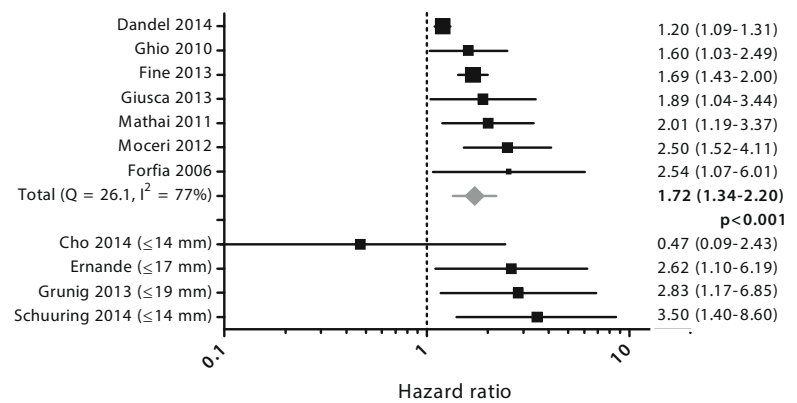

Tei index (per 0.1 increase)

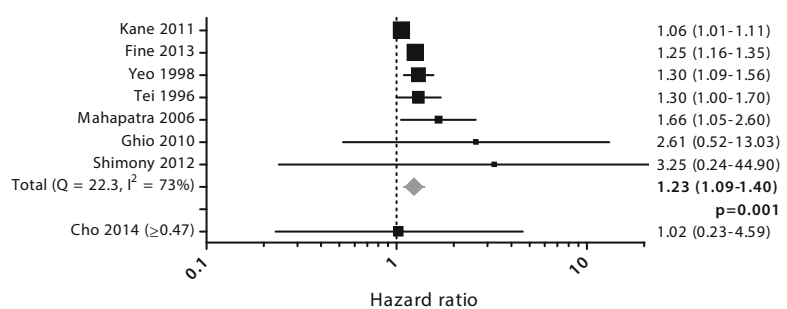

Right atrial area (per $5 \mathrm{~cm}^{2}$ increase)

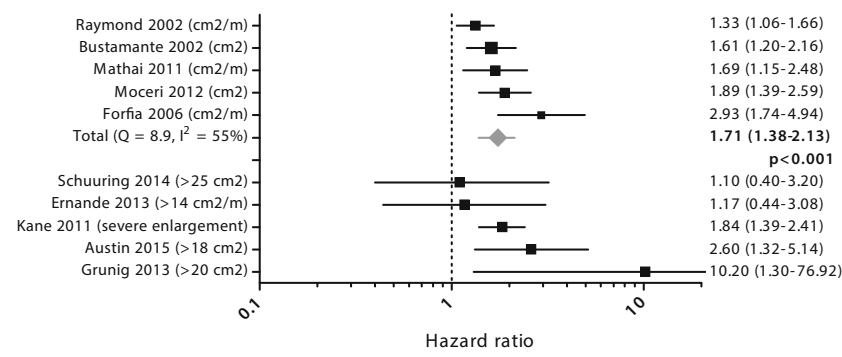

TR severity

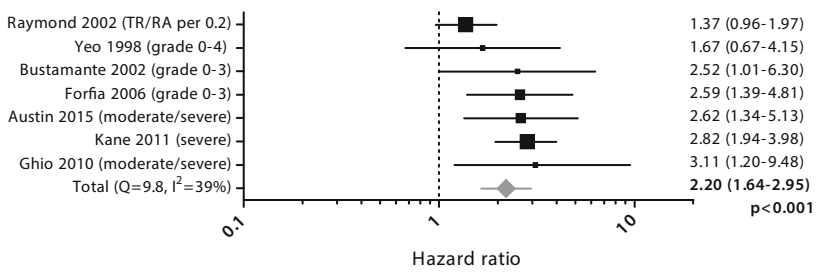

LV eccentricity index

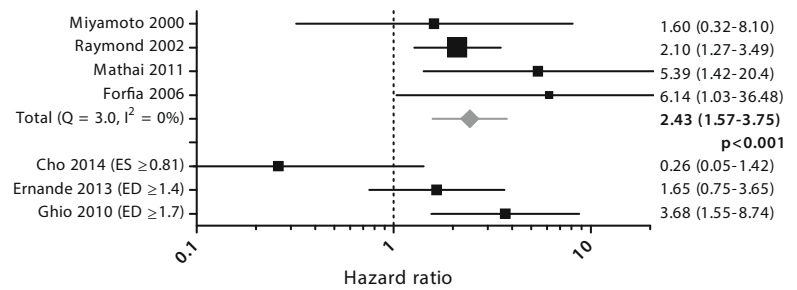

RV fractional area change (per 5\% decrease)

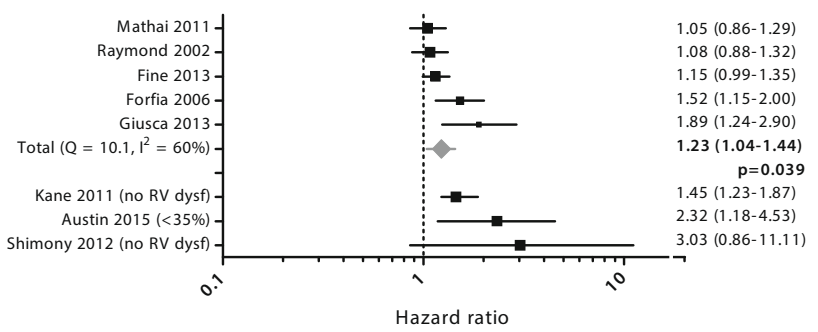

RV free wall longitudinal peak systolic strain (per $5 \%$ decrease)

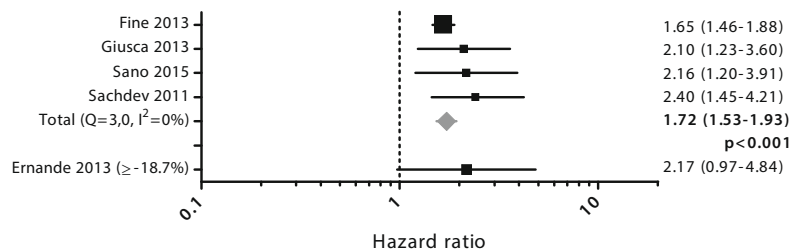

b

Fig. 3 a Prognostic value of echocardiographic findings investigated in four or more studies. b Prognostic value of echocardiographic measurements of RV function investigated in four or more studies 


\section{KEY MESSAGE}

Echocardiography is useful for prognostication in pulmonary arterial hypertension (PAH). Presence of pericardial effusion, lower TAPSE and enlarged right atrial area are the most robust predictors of mortality or transplant. This is important, since accurate prognostication can aid in adequate expansion of PAH-specific therapy and timely listing for transplantation.

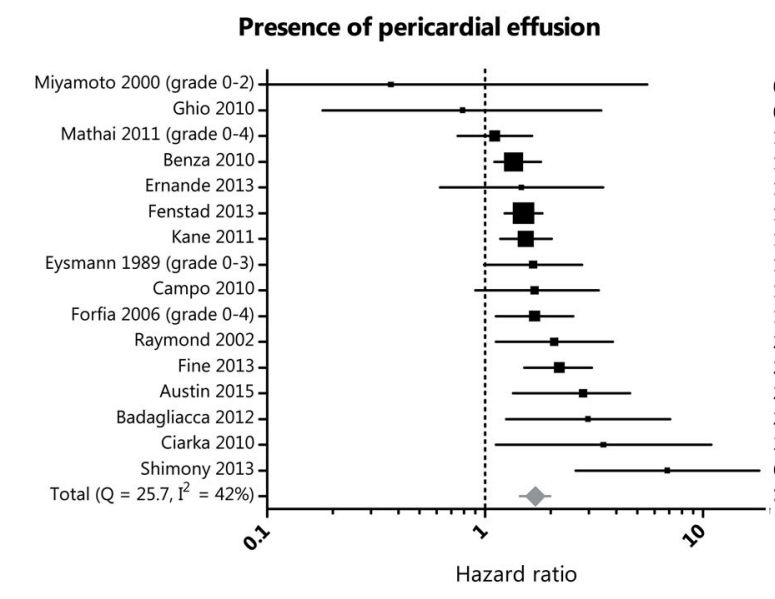

TAPSE (per $5 \mathrm{~mm}$ decrease)

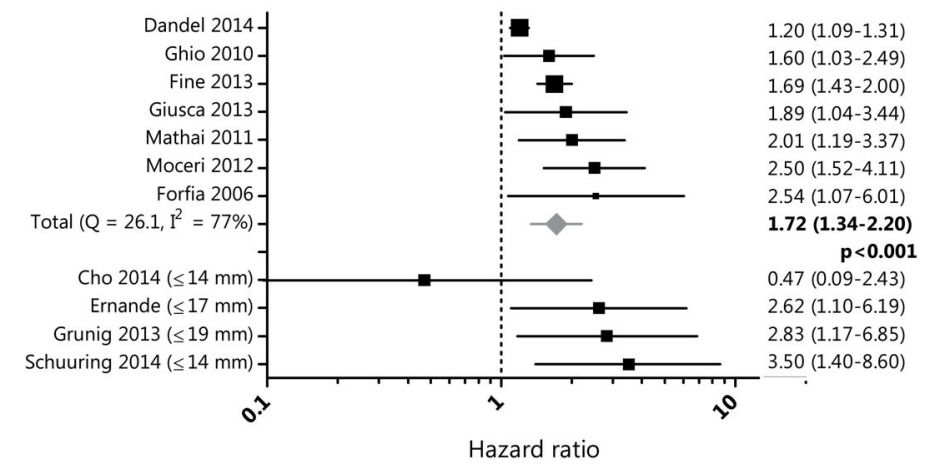

Right atrial area (per $5 \mathrm{~cm}^{2}$ increase)

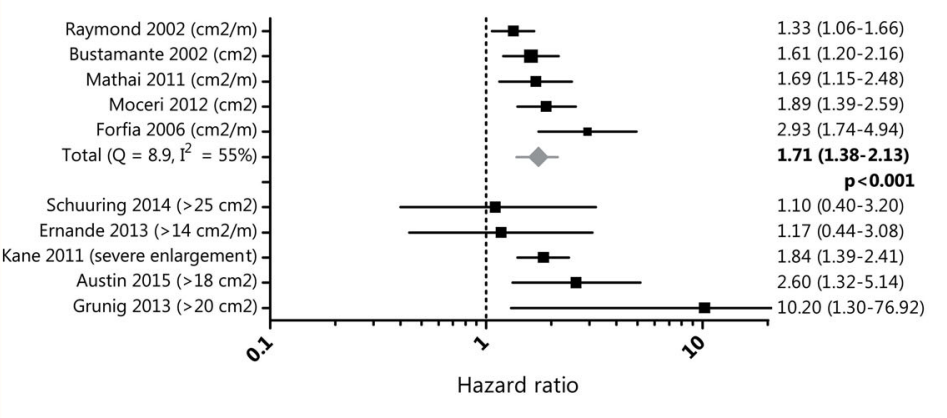

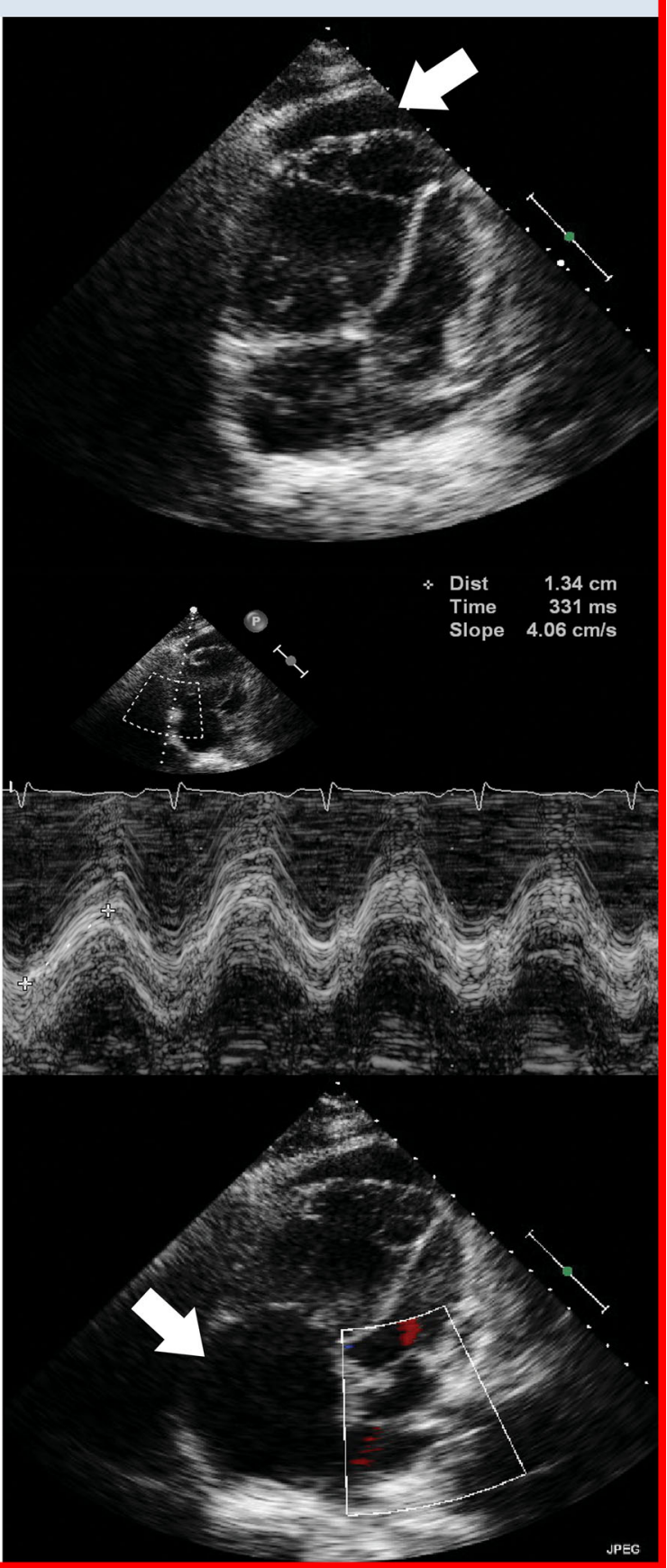

Fig. 4 Prognostic value of pericardial effusion, TAPSE and right atrial area 
Tab. 2 Sensitivity analysis for all echocardiographic measurements with significant statistical heterogeneity $\left(\mathrm{I}^{2}>50 \%\right.$ or $p$-value $\left.<0.10\right)$ in specific study subgroups

\begin{tabular}{|c|c|c|c|c|c|c|}
\hline & $\begin{array}{l}\text { No. of } \\
\text { studies }\end{array}$ & HR & $95 \% \mathrm{CI}$ & $p$-value & $\mathrm{I} 2, \%$ & $\begin{array}{l}\text { Cochran's Q } \\
\text { ( } p \text {-value) }\end{array}$ \\
\hline Presence of pericardial effusion & 16 & 1.70 & $1.44-1.99$ & $<0.001$ & 42 & $25.7(0.041)$ \\
\hline$>70 \%$ NYHA class III-IV & 8 & 1.56 & $1.31-1.86$ & $<0.001$ & 23 & $9.0(0.249)$ \\
\hline $100 \% \mathrm{PAH}$ & 12 & 1.62 & $1.34-1.96$ & $<0.001$ & 45 & $20.1(0.045)$ \\
\hline Exclusion of CHD & 12 & 1.81 & $1.45-2.25$ & $<0.001$ & 49 & $21.5(0.028)$ \\
\hline$>50 \%$ on $\mathrm{PAH}$ medication/NR & 10 & 1.86 & $1.40-2.47$ & $<0.001$ & 60 & $22.3(0.008)$ \\
\hline Mortality/transplant as outcome & 15 & 1.64 & $1.39-1.94$ & $<0.001$ & 38 & $22.5(0.069)$ \\
\hline Right atrial area, per $5 \mathrm{~cm}^{2}$ increase & 5 & 1.71 & $1.38-2.13$ & $<0.001$ & 55 & $8.9(0.063)$ \\
\hline$>70 \%$ NYHA class III-IV & 4 & 1.69 & $1.29-2.21$ & $<0.001$ & 61 & $7.8(0.051)$ \\
\hline $100 \%$ PAH & 4 & 1.56 & $1.33-1.84$ & $<0.001$ & 17 & $3.6(0.306)$ \\
\hline Exclusion of CHD & 4 & 1.69 & $1.29-2.21$ & $<0.001$ & 61 & $7.8(0.051)$ \\
\hline$>50 \%$ on $\mathrm{PAH}$ medication/NR & 3 & 1.77 & $1.17-2.68$ & 0.007 & 74 & $7.7(0.021)$ \\
\hline Mortality/transplant as outcome & 5 & 1.71 & $1.38-2.13$ & $<0.001$ & 55 & $8.9(0.063)$ \\
\hline RV pressure, per $10 \mathrm{mmHg}$ increase & 7 & 1.18 & 1.01-1.38 & 0.043 & 76 & $25.1(<0.001)$ \\
\hline$>70 \%$ NYHA class III-IV & 4 & 1.33 & $1.00-1.77$ & NS & 72 & $10.9(0.012)$ \\
\hline $100 \%$ PAH & 5 & 1.20 & $0.95-1.52$ & NS & 75 & $15.8(0.003)$ \\
\hline Exclusion of CHD & 5 & 1.26 & $1.00-1.59$ & NS & 81 & $20.6(<0.001)$ \\
\hline$>50 \%$ on $\mathrm{PAH}$ medication/NR & 5 & 1.19 & $1.02-1.38$ & 0.024 & 66 & $11.8(0.019)$ \\
\hline Mortality/transplant as outcome & 5 & 1.20 & $0.95-1.52$ & NS & 75 & $15.8(0.003)$ \\
\hline Right atrial pressure, $>15 \mathrm{mmHg}$ & 6 & 2.45 & $1.56-3.85$ & $<0.001$ & 76 & $20.7(<0.001)$ \\
\hline$>70 \%$ NYHA class III-IV & 2 & 1.38 & $0.65-2.92$ & NS & 82 & $5.7(0.017)$ \\
\hline $100 \%$ PAH & 6 & 2.45 & $1.56-3.85$ & $<0.001$ & 76 & $20.7(<0.001)$ \\
\hline Exclusion of CHD & 4 & 2.41 & $1.16-4.98$ & 0.018 & 82 & $17.0(<0.001)$ \\
\hline$>50 \%$ on $\mathrm{PAH}$ medication/NR & 0 & - & - & - & - & - \\
\hline Mortality/transplant as outcome & 5 & 2.28 & $1.33-3.92$ & 0.003 & 72 & $14.2(0.007)$ \\
\hline TAPSE, per $5 \mathrm{~mm}$ decrease & 7 & 1.72 & $1.34-2.20$ & $<0.001$ & 77 & $26.1(<0.001)$ \\
\hline$>70 \%$ NYHA class III-IV & 3 & 1.63 & $1.01-2.63$ & 0.047 & 69 & $6.5(0.039)$ \\
\hline $100 \%$ PAH & 4 & 1.67 & $1.15-2.44$ & 0.007 & 77 & $12.8(0.005)$ \\
\hline Exclusion of CHD & 5 & 1.58 & $1.22-2.06$ & $<0.001$ & 79 & $18.7(<0.001)$ \\
\hline$>50 \%$ on $\mathrm{PAH}$ medication/NR & 4 & 3.24 & $1.92-5.45$ & $<0.001$ & 0 & $1.2(0.756)$ \\
\hline Mortality/transplant as outcome & 5 & 1.76 & $1.22-2.52$ & 0.002 & 74 & $15.4(0.004)$ \\
\hline RV FAC, per $5 \%$ decrease & 5 & 1.23 & $1.04-1.44$ & $\mathbf{0 . 0 3 9}$ & 60 & $10.1(0.039)$ \\
\hline$>70 \%$ NYHA class III-IV & 3 & 1.18 & $0.96-1.44$ & NS & 60 & $5.0(0.080)$ \\
\hline $100 \%$ PAH & 2 & 1.06 & $1.04-1.09$ & $<0.001$ & 0 & $0.0(0.863)$ \\
\hline Exclusion of CHD & 4 & 1.16 & $1.02-1.32$ & 0.026 & 41 & $5.0(0.168)$ \\
\hline$>50 \%$ on $\mathrm{PAH}$ medication/NR & 5 & 1.23 & $1.04-1.44$ & 0.039 & 60 & $10.1(0.039)$ \\
\hline Mortality/transplant as outcome & 3 & 1.18 & $0.96-1.44$ & NS & 60 & $5.0(0.080)$ \\
\hline Tei index, per 0.1 unit increase & 7 & 1.23 & $1.09-1.40$ & 0.001 & 73 & $22.3(0.001)$ \\
\hline$>70 \%$ NYHA class III-IV & 3 & 1.22 & $0.99-1.51$ & NS & 76 & $8.3(0.016)$ \\
\hline $100 \%$ PAH & 6 & 1.25 & $1.05-1.48$ & 0.012 & 58 & $12.0(0.035)$ \\
\hline Exclusion of CHD & 5 & 1.46 & $1.24-1.72$ & $<0.001$ & 0 & $2.3(0.677)$ \\
\hline$>50 \%$ on $\mathrm{PAH}$ medication/NR & 2 & 1.25 & $1.16-1.35$ & $<0.001$ & 0 & $0.5(0.775)$ \\
\hline Mortality/transplant as outcome & 6 & 1.25 & $1.05-1.48$ & 0.012 & 58 & $12.0(0.035)$ \\
\hline
\end{tabular}

$C H D$ congenital heart disease, $C I$ confidence interval, FAC fractional area change, $H R$ hazard ratio, NR not reported, NS non-significant, NYHA New York Heart Association, $P A H$ pulmonary arterial hypertension, $R V$ right ventricular, TAPSE tricuspid annular plane systolic excursion 
plant used) to investigate whether this impacted the pooled HR. For presence of pericardial effusion, right atrial area and TAPSE, sensitivity analysis did not change the overall conclusions. These results can therefore be regarded with a higher degree of certainty (Fig. 4).

No sensitivity analysis was performed for severity of tricuspid regurgitation, LV eccentricity index and RV free wall LPSS; however the forest plots show that especially tricuspid regurgitation severity and LV eccentricity index have relatively large standard errors, and thus provide imprecise risk estimations.

A combination of visual assessment of funnel plots and Egger's test provided statistical evidence of publication bias for TAPSE $(p=0.026)$, right atrial area $(p=0.027)$ and the Tei index $(p=0.076$ and based on the funnel plot). This may indicate that studies with a positive result are overrepresented, subsequently leading to a relative overestimation of the pooled HR in the meta-analysis.

\section{Discussion}

To our knowledge, this is the first systematic review and meta-analysis on the prognostic value of specific echocardiographic findings in patients with PAH. Among 51 echocardiographic findings investigated in 37 studies, meta-analysis and additional sensitivity analysis showed that presence of pericardial effusion, right atrial area and TAPSE were the most robust predictors of mortality or transplant in patients with PAH.

\section{Right ventricular decompensation}

Most deaths in patients with PAH are due to right heart failure [7]. Once the right ventricle starts to fail, it is no longer able to overcome the high pulmonary arterial pressures. This will cause a progressive rise in RV diastolic pressure and right atrial pressure, generally accompanied by right atrial enlargement. It is thought that elevated right atrial pressure causes impaired lymphatic and venous drainage, subsequently leading to pericardial fluid accumulation [17]. These insights into the mechanistic course tilting a stable PAH state towards death clearly explain why pericardial effusion, right atrial area, estimated right atrial pressure and $\mathrm{RV}$ dysfunction measured on echocardiography are associated with mortality in PAH.

Studies investigating RV function and right atrial pressure as assessed with other diagnostic modalities, such as cardiac magnetic resonance imaging and right heart catheterisation, report comparable findings [6, 7, 48, 49]. However, as echocardiography is more widely applied, non-invasive and less expensive, it is more suitable for the evaluation of PAH patients in day-to-day practice.

\section{Serial measurements}

The majority of the included studies investigated the prognostic value of baseline imaging findings, evaluated at the time of diagnosis. Complementary information on changes in haemodynamic, functional and biochemical variables may better reflect an individual's response to PAH-targeted therapy - or progression of disease [50]. Interestingly, although the first study included in this review originates from 1989, serial echocardiographic evaluation in PAH has only recently gained scientific attention, as Hardegree and colleagues were the first ones to publish on this topic in $2013[12,39]$. Thus far, changes in pericardial fluid accumulation, right atrial area, tricuspid regurgitation velocity, RV free wall LPSS and qualitative RV function, RV dimensions and RV load-adaptation index have been associated with clinical outcomes in PAH [39, 42, 44, 46, 47]. This is in line with the conclusions reached by the international working group of Vonk-Noordegraaf et al., who stated that changes in RV imaging parameters after treatment reflect altered exercise capacity and predict subsequent survival [51]. Advantages of echocardiography over more expensive or invasive imaging modalities become especially important in the serial evaluation of individual patients.

\section{Heterogeneity}

PAH prognosis depends largely on the underlying aetiology, as the right ventricle can show rapid deterioration after initial diagnosis in patients with idiopathic or connective tissue disease-PAH, while it may cope successfully with pressure overload for decades in patients with congenital heart disease $[4,6]$. Still, most studies in this review investigated the World Health Organisation (WHO) group I PAH as a whole. Moreover, some studies in this review additionally included small subsets $(<30 \%)$ of patients with WHO group III (pulmonary hypertension due to lung disease) or IV (chronic thromboembolic pulmonary hypertension), which further increases the heterogeneity of the study population. The heterogeneity of pulmonary hypertension aetiologies among studies in this review likely plays a major role in the observed variation between the reported study results. Along the same line, disease severity and changing available treatment options over time likely contribute to this observed heterogeneity across studies. We therefore conducted a sensitivity analysis in which we excluded studies with other than WHO group I PAH, patients with CHD, $<70 \%$ patients in NYHA class III-IV (thus investigating a sicker patient population) or $<50 \%$ on PAH medication (representing other available treatment options). Importantly, this clearly reduced the statistical heterogeneity in specific study subgroups; however the overall conclu- 
sions for presence of pericardial effusion, right atrial area and TAPSE remained unchanged.

\section{Study limitations}

We presented only univariable HRs in this study, because of the large variety between studies in which multivariable adjustment was performed (regarding the type and number of predictors per event used). Second, formal tests for publication bias retrieved significant results for the variables right atrial area, TAPSE and Tei index. Theoretically, publication bias may cause underreporting of non-significant HRs, leading to a relative overestimation of the pooled HRs. Exact results of the random effect models as presented in this review should be therefore interpreted with caution.

\section{Clinical implications}

In order to adequately expand PAH-specific therapy and timely list patients for transplantation, accurate prognostication is highly important. The data in this review imply that especially pericardial effusion, enlarged right atrial area and decreased TAPSE are useful echocardiographic markers to predict mortality or transplantation. This is largely concordant with the 2015 European Society of Cardiology/ European Respiratory Society Guidelines for the diagnosis and treatment of pulmonary hypertension, in which right atrial area and pericardial effusion are recommended as determinants of prognosis [2]. Controversy continues to exist about the use of TAPSE; it has been suggested that progressive RV dysfunction is associated with a decline in TAPSE until a certain floor effect is reached [52]. Of note, considering the multi-faceted nature of this disease, accurate prognostication should always be based on a combination of haemodynamic, functional, biochemical and echocardiographic findings, and should not rely on just one single parameter according to the current guidelines [2].

\section{Conclusions}

This meta-analysis substantiates the clinical yield of specific echocardiographic findings in the prognostication of PAH patients in day-to-day practice. Although accurate prognostication should not rely on just one single parameter, presence of pericardial effusion, enlarged right atrial area and decreased TAPSE are the most firmly established echocardiographic tools that can be of important additional value.

Conflict of interest V.J.M. Baggen, M.M.P. Driessen, M.C. Post, A.P. van Dijk, J.W. Roos-Hesselink, A.E. van den Bosch, J.J.M. Takkenberg and G.T. Sieswerda state that there are no conflicts of interest.
Open Access This article is distributed under the terms of the Creative Commons Attribution 4.0 International License (http:// creativecommons.org/licenses/by/4.0/), which permits unrestricted use, distribution, and reproduction in any medium, provided you give appropriate credit to the original author(s) and the source, provide a link to the Creative Commons license, and indicate if changes were made.

\section{References}

1. Simonneau G, Gatzoulis MA, Adatia I, et al. Updated clinical classification of pulmonary hypertension. J Am Coll Cardiol. 2013;62:D34-41.

2. Galie N, Humbert M, Vachiery JL, et al. ESC/ERS Guidelines for the diagnosis and treatment of pulmonary hypertension: The Joint Task Force for the Diagnosis and Treatment of Pulmonary Hypertension of the European Society of Cardiology (ESC) and the European Respiratory Society (ERS): Endorsed by: Association for European Paediatric and Congenital Cardiology (AEPC), International Society for Heart and Lung Transplantation (ISHLT). Eur Respir J. 2015;2015(46):903-75.

3. Humbert M, Lau EM, Montani D, Jais X, Sitbon O, Simonneau G. Advances in therapeutic interventions for patients with pulmonary arterial hypertension. Circulation. 2014;130:2189-208.

4. McLaughlin VV, Presberg KW, Doyle RL, et al. Prognosis of pulmonary arterial hypertension. Chest. 2004;126:78S-92S.

5. Humbert M, Sitbon O, Chaouat A, et al. Survival in patients with idiopathic, familial, and anorexigen-associated pulmonary arterial hypertension in the modern management era. Circulation. 2010;122:156-63.

6. Benza RL, Miller DP, Gomberg-Maitland M, et al. Predicting survival in pulmonary arterial hypertension: insights from the Registry to Evaluate Early and Long-Term Pulmonary Arterial Hypertension Disease Management (REVEAL). Circulation. 2010;122:164:72.

7. D'Alonzo GE, Barst RJ, Ayres SM, et al. Survival in patients with primary pulmonary hypertension. Results from a national prospective registry. Ann Intern Med. 1991;115:343-9.

8. Blok IM, Riel AC van, Schuuring MJ, et al. Decrease in quality of life predicts mortality in adult patients with pulmonary arterial hypertension due to congenital heart disease. Neth Heart J. 2015;23:278-84.

9. Moher D, Liberati A, Tetzlaff J, Altman DG, Group P. Preferred reporting items for systematic reviews and meta-analyses: the PRISMA statement. Ann Intern Med. 2009;151(W64):264-9.

10. Geersing GJ, Bouwmeester W, Zuithoff P, Spijker R, Leeflang M, Moons K. Search filters for finding prognostic and diagnostic prediction studies in Medline to enhance systematic reviews. PLoS ONE. 2012;7:e32844.

11. Hayden JA, Cote P, Bombardier C. Evaluation of the quality of prognosis studies in systematic reviews. Ann Intern Med. 2006; 144:427-37.

12. Eysmann SB, Palevsky HI, Reichek N, Hackney K, Douglas PS. Two-dimensional and Doppler-echocardiographic and cardiac catheterization correlates of survival in primary pulmonary hypertension. Circulation. 1989;80:353-60.

13. Tei C, Dujardin KS, Hodge DO, et al. Doppler echocardiographic index for assessment of global right ventricular function. J Am Soc Echocardiogr. 1996;9:838-47.

14. Yeo TC, Dujardin KS, Tei C, Mahoney DW, McGoon MD, Seward JB. Value of a Doppler-derived index combining systolic and diastolic time intervals in predicting outcome in primary pulmonary hypertension. Am J Cardiol. 1998;81:1157-61.

15. Miyamoto S, Nagaya N, Satoh T, et al. Clinical correlates and prognostic significance of six-minute walk test in patients with primary 
pulmonary hypertension: Comparison with cardiopulmonary exercise testing. Am J Respir Crit Care Med. 2000;161:487-92.

16. Bustamante-Labarta MH, Perrone S. Leon de la Fuente R, et al. Right atrial size and tricuspid regurgitation severity predict mortality or transplantation in primary pulmonary hypertension. J Am Soc Echocardiogr. 2002;15:1160-4.

17. Raymond RJ, Hinderliter AL, Willis PW, et al. Echocardiographic predictors of adverse outcomes in primary pulmonary hypertension. J Am Coll Cardiol. 2002;39:1214-9.

18. Forfia PR, Fisher MR, Mathai SC, et al. Tricuspid annular displacement predicts survival in pulmonary hypertension. Am J Respir Crit Care Med. 2006;174:1034-41.

19. Mahapatra S, Nishimura RA, Oh JK, McGoon MD. The prognostic value of pulmonary vascular capacitance determined by doppler echocardiography in patients with pulmonary arterial hypertension. J Am Soc Echocardiogr. 2006;19:1045-50.

20. Utsunomiya H, Nakatani S, Nishihira M, et al. Value of estimated right ventricular filling pressure in predicting cardiac events in chronic pulmonary arterial hypertension. J Am Soc Echocardiogr. 2009;22:1368-74.

21. Campo A, Mathai SC, Pavec J Le, et al. Hemodynamic predictors of survival in scleroderma-related pulmonary arterial hypertension. Am J Respir Crit Care Med. 2010;182:252-60.

22. Ciarka A, Doan V, Velez-Roa S, Naeije R, Borne P Van De. Prognostic significance of sympathetic nervous system activation in pulmonary arterial hypertension. Am J Respir Crit Care Med. 2010;181:1269-75.

23. Ghio S, Klersy C, Magrini G, et al. Prognostic relevance of the echocardiographic assessment of right ventricular function in patients with idiopathic pulmonary arterial hypertension. Int J Cardiol. 2010;140:272-8.

24. Ghio S, Pazzano AS, Klersy C, et al. Clinical and prognostic relevance of echocardiographic evaluation of right ventricular geometry in patients with idiopathic pulmonary arterial hypertension. Am J Cardiol. 2011;107:628-32.

25. Kane GC, Maradit-Kremers H, Slusser JP, Scott CG, Frantz RP, McGoon MD. Integration of clinical and hemodynamic parameters in the prediction of long-term survival in patients with pulmonary arterial hypertension. Chest. 2011;139:1285-93.

26. Mathai SC, Sibley CT, Forfia PR, et al. Tricuspid annular plane systolic excursion is a robust outcome measure in systemic sclerosis-associated pulmonary arterial hypertension. J Rheumatol. 2011;38:2410-8.

27. Sachdev A, Villarraga HR, Frantz RP, et al. Right ventricular strain for prediction of survival in patients with pulmonary arterial hypertension. Chest. 2011;139:1299-309.

28. Zeng WJ, Sun YJ, Xiong CM, Gu Q, He JG. Prognostic value of echocardiographic right/left ventricular end-diastolic diameter ratio in idiopathic pulmonary arterial hypertension. Chin Med J. 2011;124:1672-77.

29. Badagliacca R, Pezzuto B, Poscia R, et al. Prognostic factors in severe pulmonary hypertension patients who need parenteral prostanoid therapy: The impact of late referral. J Heart Lung Transplant. 2012;31:364-72.

30. Moceri P, Dimopoulos K, Liodakis E, et al. Echocardiographic predictors of outcome in eisenmenger syndrome. Circulation. 2012;126:1461-8.

31. Shimony A, Fox BD, Afilalo J, Rudski LG, Hirsch A, Langleben D. Pulmonary arterial hypertension in the elderly-clinical characteristics and long-term survival. Lung. 2012;190:645-9.

32. Tonelli AR, Plana JC, Heresi GA, Dweik RA. Prevalence and prognostic value of left ventricular diastolic dysfunction in idiopathic and heritable pulmonary arterial hypertension. Chest. 2012;141:1457-65.

33. Ernande L, Cottin V, Leroux PY, et al. Right isovolumic contraction velocity predicts survival in pulmonary hypertension. J Am Soc Echocardiogr. 2013;26:297-306.
34. Fenstad ER, Le RJ, Sinak LJ, et al. Pericardial effusions in pulmonary arterial hypertension: characteristics, prognosis, and role of drainage. Chest. 2013;144:1530-8.

35. Fine NM, Chen L, Bastiansen PM, et al. Outcome prediction by quantitative right ventricular function assessment in 575 subjects evaluated for pulmonary hypertension. Circ Cardiovasc Imaging. 2013;6:711-21.

36. Giusca S, Jurcut R, Coman IM, et al. Right ventricular function predicts clinical response to specific vasodilator therapy in patients with pulmonary hypertension. Echocardiography. 2013;30:17-26.

37. Grunig E, Tiede H, Enyimayew EO, et al. Assessment and prognostic relevance of right ventricular contractile reserve in patients with severe pulmonary hypertension. Circulation. 2013;128:2005-15.

38. Hardegree EL, Sachdev A, Fenstad ER, et al. Impaired left ventricular mechanics in pulmonary arterial hypertension identification of a cohort at high risk. Circ Heart Fail. 2013;6:748-55.

39. Hardegree EL, Sachdev A, Villarraga HR, et al. Role of serial quantitative assessment of right ventricular function by strain in pulmonary arterial hypertension. Am J Cardiol. 2013;111:143-8.

40. Shimony A, Fox BD, Langleben D, Rudski LG. Incidence and significance of pericardial effusion in patients with pulmonary arterial hypertension. Can J Cardiol. 2013;29:678-82.

41. Cho IJ, Oh J, Chang HJ, et al. Tricuspid regurgitation duration correlates with cardiovascular magnetic resonance-derived right ventricular ejection fraction and predict prognosis in patients with pulmonary arterial hypertension. Eur Heart J Cardiovasc Imaging. 2014;15:18-23.

42. Tonelli AR, Conci D, Tamarappoo BK, Newman J, Dweik RA. Prognostic value of echocardiographic changes in patients with pulmonary arterial hypertension receiving parenteral prostacyclin therapy. J Am Soc Echocardiogr. 2014;27:733-41:e2.

43. Schuuring MJ, Riel ACMJ van, Vis JC, et al. New predictors of mortality in adults with congenital heart disease and pulmonary hypertension: Midterm outcome of a prospective study. Int J Cardiol. 2015;181:270-6.

44. Dandel M, Knosalla C, Kemper D, Stein J, Hetzer R. Assessment of right ventricular adaptability to loading conditions can improve the timing of listing to transplantation in patients with pulmonary arterial hypertension. J Heart Lung Transplant. 2015;34:319-28.

45. Austin C, Alassas K, Burger C, et al. Echocardiographic assessment of estimated right atrial pressure and size predicts mortality in pulmonary arterial hypertension. Chest. 2015;147:198-208.

46. Batal O, Dardari Z, Costabile C, Gorcsan J, Arena VC, Mathier MA. Prognostic value of pericardial effusion on serial echocardiograms in pulmonary arterial hypertension. Echocardiography. 2015;32:1471-6.

47. Sano H, Tanaka H, Motoji Y, et al. Right ventricular function and right-heart echocardiographic response to therapy predict long-term outcome in patients with pulmonary hypertension. Can J Cardiol. 2015;31:529-36.

48. Wolferen SA van, Marcus JT, Boonstra A, et al. Prognostic value of right ventricular mass, volume, and function in idiopathic pulmonary arterial hypertension. Eur Heart J. 2007;28:1250-7.

49. Veerdonk MC van de, Kind T, Marcus JT, et al. Progressive right ventricular dysfunction in patients with pulmonary arterial hypertension responding to therapy. J Am Coll Cardiol. 2011;58:2511-9.

50. Nickel N, Golpon H, Greer M, et al. The prognostic impact of follow-up assessments in patients with idiopathic pulmonary arterial hypertension. Eur Respir J. 2012;39:589-96.

51. Vonk Noordegraaf A, Haddad F, Bogaard HJ, Hassoun PM. Noninvasive imaging in the assessment of the cardiopulmonary vascular unit. Circulation. 2015;131:899-913.

52. Mauritz GJ, Kind T, Marcus JT, et al. Progressive changes in right ventricular geometric shortening and long-term survival in pulmonary arterial hypertension. Chest. 2012;141:935-43. 


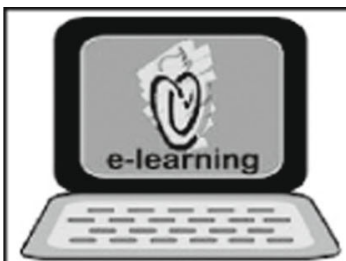

\section{CVOI E-learning formula!}

This is the CVOI e-learning article. The author has prepared 10 questions which are available through the website of the Cardiovascular Educational Institute (CVOI). Please follow the instructions below.

After finishing the questions you will be asked to fill in your name, hospital and e-mail address; then press the button 'verzenden'.

When 6 out of the 10 questions are answered correctly, you acquire 1 accreditation point granted by the Quality Committee of the Netherlands Society of Cardiology (NVVC). The acquired point will be credited to your personal file in the GAIA system. You will also receive an e-mail with all the correct answers.

Over a period of one year 10 e-learning articles will appear in 10 subsequent NHJ editions. In each edition the e-learning article will be recognisable by a special icon. On an annual basis you can collect 10 accreditation points. The accreditation points are credited in the GAIA system by the CVOI.

If you need additional information, please contact the CVOI by e-mail: cvoi@cvoi.org or by phone: 030-2345001.

E.E. van der Wall

Chief editor NHJ
K.B. Schick

Coordinator CVOI 